\title{
CONSULTAS EN TORNO A TEMAS DE CRIANZA Y SU RELACIÓN CON EL APRENDIZAJE DE LA CONVIVENCIA \\ CONCERNS ABOUT TOPICS ON UPBRINGING AND THEIR RELATION TO THE LEARNING PROCESS OF COHABITATION
}

\author{
Volumen 14, Número 2 \\ Mayo - Agosto \\ pp. 1-23
}

Este número se publicó el 30 de mayo de 2014

Ana Rocío Barquero Brenes

Revista indizada en REDALYC, SCIELO

Revista distribuida en las bases de datos:

CATÁLOGO DE LATINDEX, IRESIE, CLASE, DIALNET, DOAJ, E-REVIST@S, SHERPA/ROMEO, QUALIS, MIAR

Revista registrada en los directorios:

ULRICH'S, REDIE, RINACE, OEI, MAESTROTECA, PREAL, CLASCO 


\title{
CONSULTAS EN TORNO A TEMAS DE CRIANZA Y SU RELACIÓN CON EL APRENDIZAJE DE LA CONVIVENCIA \\ CONCERNS ABOUT TOPICS ON UPBRINGING AND THEIR RELATION TO THE LEARNING PROCESS OF COHABITATION
}

\begin{abstract}
Ana Rocío Barquero Brenes ${ }^{1}$
Resumen: En el presente artículo se sistematizaron inquietudes en torno a temas de crianza, recopiladas por el Departamento de Psicología de un centro educativo, ubicado en el área metropolitana en Costa Rica, durante los ciclos lectivos 2010 y 2011. Se seleccionó un enfoque mixto, que implicó un proceso de recolección, análisis y vinculación de datos cuantitativos y cualitativos. Uno de los intereses más importantes, fue indagar si las inquietudes y estrategias empleadas por las familias en la crianza, eran o no compatibles con el aprendizaje de la convivencia. En lo que respecta a los principales resultados, se evidenciaron cambios en la estructura y dinámica de las familias, que inciden directamente en las competencias para la crianza y en la promoción de la convivencia. Las diversas demandas, presiones sociales y multiplicidad de tareas interfieren en la cohesión grupal, menoscabando la función socializadora y, específicamente, el aprendizaje de la convivencia.
\end{abstract}

Palabras clave: CONVIVENCIA, FAMILIA, CRIANZA, PSICOLOGÍA EDUCATIVA, COSTA RICA.

Abstract: This article systematizes the concerns around the topic of upbringing and the learning process of cohabitation gathered by the Department of Psychology of an educational center located in the Great Metropolitan Area, in Costa Rica, between the years 2010 and 2011. A mixed approach consisting on a process of recollection, analysis and connection of both quantitative and qualitative data was selected. One of the main interests was to investigate if the concerns and strategies used by the families were compatible with the learning process of cohabitation. Regarding the main results, changes in the structure and dynamic of the families were evidenced and these influenced directly parenting skills and therefore the learning process to achieve a better cohabitation. The various demands, social pressures and multiplicity of functions (that must be handed by the adults) interfere with the group cohesion, taking away importance from the socializing function and specifically the learning process of cohabitation.

Key words: COHABITATION, FAMLILY, RAISING CHILDREN, EDUCATIONAL PSYCHOLOGY, COSTA RICA.

\footnotetext{
1 Docente en la Escuela de Psicología de la Universidad de Costa Rica, también labora en un centro educativo. Máster en Psicología Educativa, Universidad de Costa Rica, San José, Costa Rica.
}

Dirección electrónica: ana.barquero@ucr.ac.cr

Artículo recibido: 25 de julio, 2013

Devuelto para corrección: $1^{\circ}$ de noviembre, 2013

Aprobado: 12 de diciembre, 2013 


\section{Introducción}

La familia es una categoría histórica, marcada por aspectos políticos, económicos y culturales. De acuerdo con Espinosa (2009), es el campo de las relaciones afectivas más profundas y, por lo tanto, constituye uno de los pilares de la identidad personal; todas las dimensiones humanas más significativas se plasman y transmiten en la cotidianidad de la vida familiar. Para la autora Arès (2009), la familia constituye un sistema de relaciones que es más que la suma de sus miembros, donde se cumplen funciones básicas, económicas, biológicas, espirituales, culturales y educativas.

En el seno de la familia se instaura el proceso de socialización, ya que es el primer grupo humano que ejerce la intermediación individuo-sociedad. Al respecto, indica Rodríguez (2006) que la familia es una institución mediadora de la realidad en el proceso de integración del ser humano a su comunidad. Por tanto, tiene por delante el reto de encauzar a sus integrantes, para que adquieran y desarrollen habilidades sociales que les permitan manejar adecuadamente sus emociones, comprender su contexto y ubicarse como proactivos en la lucha por la construcción de una cultura de paz.

Este reto al que se refiere Rodríguez, se ve empañado por los desafíos que enfrenta la familia actual. Para Murueta (2009), la familia vigente es una compleja multiplicidad de tipologías producto de la globalización, que atraviesa por grandes presiones sociales. Los intereses de índole económica conducen a que padres y madres asuman muchas funciones simultáneas, e inviertan gran parte del tiempo en tareas fuera del espacio familiar. Como consecuencia, cuando tienen lugar las interacciones, predominan el cansancio y la culpa, que a su vez conlleva a los abusos, el irrespeto y la violencia.

Al respecto, indica Espinosa (2009) que la familia actual está sometida a altos niveles de estrés, debido a las demandas y presiones sociales a las que está sometida; esto genera que progenitores se sientan confundidos, infelices, ineficaces y desorientados frente al ejercicio de la crianza.

De lo expuesto se desprende que la función básica de la familia como instancia formadora, ha venido en detrimento, perdiendo el protagonismo que la identificaba décadas atrás. El Ministerio de Educación de Colombia (2005) refiere que la familia actual ha traslado a la escuela la formación ciudadana. Afirma que en el núcleo familiar se han dejado de promover competencias que generan prácticas proclives a la convivencia. 
Producto de estos planteamientos y de la indagación bibliográfica sobre la temática, se definieron los objetivos de la presente investigación. Estos se orientaron, principalmente, a indagar sobre las inquietudes y estrategias empleadas por las familias en la crianza, y si estas eran compatibles con educar la convivencia. En el entendido que los aprendizajes que se adquieren en la infancia, pueden generalizarse posteriormente a otras instancias sociales. Ante todo, es importante considerar que la promoción de la convivencia implica la capacidad de las personas de vivir con otras (con-vivir) en un marco de respeto mutuo y solidaridad recíproca, partiendo del reconocimiento y respeto por la diversidad, y de la capacidad de las personas de entenderse, valorar y aceptar las diferencias (Ministerio de Educación de Chile, 2008).

Por último, en el presente estudio se eligió un diseño mixto y la fuente de información se obtuvo a partir del registro de 226 formularios de consultas sobre temas de crianza. Las inquietudes fueron formuladas por personas a cargo de población infantil (padres, madres, tías, abuelos y abuelas) al Departamento de Psicología de un centro educativo ubicado en Costa Rica, en el área metropolitana, durante los ciclos lectivos 2010 y 2011 . En lo que respecta al análisis cuantitativo y cualitativo de los datos, las consultas se sistematizaron en preguntas y, posteriormente, en categorías.

\section{Referente teórico}

\subsection{Familia como instancia socializadora}

El estudio de la familia ha sido abordado por distintos campos científicos, según el interés particular de cada uno de ellos. La filosofía, la sociología, el derecho, la religión y la psicología, por nombrar algunos, han brindado una definición del concepto con diferentes grados de alcance social, académico y científico.

En el área psicosocial, la profesora e investigadora cubana, Arès Muzio, se refiere al núcleo primario como

... es el grupo de intermediación entre el individuo y la sociedad. Constituye el núcleo más primario del ser humano, en ella el hombre inscribe sus primeros sentimientos, sus primeras vivencias, incorpora las principales pautas de comportamiento y le da sentido a su vida. (Arès, 2002, p. 1) 
Asimismo, la autora plantea que la familia es la unión de personas que comparten un proyecto vital de existencia común, en el que se generan fuertes sentimientos de pertenencia, estableciéndose un compromiso personal entre sus miembros e intensas relaciones de intimidad, reciprocidad y dependencia. Adicionalmente, es un sistema de relaciones cualitativamente diferente a la simple suma de sus miembros, donde se cumplen funciones básicas como la económica, biológica, cultural-espiritual y la educativa, que constituye una supra función que se deriva del cumplimiento de las anteriores (Arès, 2002).

Otros autores, Murueta y Osorio (2009), refieren que "la familia es el primer entorno natural en donde los miembros que la forman evolucionan y se desarrollan en términos afectivos, físicos, intelectuales y sociales, según modelos vivenciados e interiorizados" (p. 14). Plantean que cada familia es un sistema abierto en continuo movimiento, cambio y reestructuración, en busca de una estabilidad y equilibrio entre todos los miembros que la componen y el entorno social en el cual viven. Además, es un sistema básico de referencias afectivas que le permite a una persona encontrar su propio significado personal, así como los significados de todo lo que le rodea y, por tanto, permite encontrar un determinado sentido a la vida y a las actividades cotidianas (Murueta y Osorio, 2009).

De acuerdo con Espinosa (2009), la familia es el campo de las relaciones afectivas más profundas y, por tanto, constituye uno de los pilares de la identidad de una persona. En su seno se instaura el proceso de socialización de la humanidad:

Allí se tejen los lazos afectivos primarios, los modos de expresar el afecto, la vivencia del tiempo y del espacio, las distancias corporales, el lenguaje, el ejercicio de la autoridad, qué tareas corresponden a cada sexo, cómo se ayuda al grupo familiar, quién provee las necesidades, qué códigos de comunicación están permitidos, ya sea gestuales, orales o el silenciamiento de emociones y sentimientos, el sentido de la vida y la muerte, la importancia de las fiestas, reuniones sociales o ceremonias, se inscribe también la historia de la familia que comprende a las distintas generaciones que nos precedieron; es decir, todas las dimensiones humanas más significativas se plasman y transmiten en la cotidianeidad de la vida en familia. (Espinosa, 2009, p.43)

\subsection{Transformación de la figura tradicional de familia}

Arès refiere que la familia es una categoría histórica, determinada por el sistema social que le sirve de marco. El modo de producción imperante condiciona las formas de existencia 
de la familia, las jerarquías de sus funciones, los valores predominantes y los principios éticos. De acuerdo con Arès (2002), "el grupo familiar ha resistido a los impactos de los cambios sociales, sin desaparecer como grupo humano, se ha diversificado en su tipología, cambiado las formas de hacer pareja, los estilos de autoridad, los modelos de paternidad y maternidad" (p.06).

Para Murueta (2009) la familia actual cuenta con menos espacio para el esparcimiento, la convivencia, la comunicación. Opina que, debido al cansancio y a las limitaciones económicas, son pocas las familias que promueven la convivencia activa. Dadas las tensiones y los vacíos emocionales que sufren padres e hijos, sus interacciones se van llenando de regaños, reclamaciones, culpas, chantajes y abusos mutuos. Esta situación se traduce, en la sociedad, en el crecimiento de abusos, irrespeto y violencia:

La disminución de la afectividad en la familia produce la falta de sensibilidad hacia el sufrimiento de los demás, e incluso éste se ve como motivo de goce morboso, la falta de empatía y la frustración personal genera recelos y envidias ante el bienestar ajeno. (Murueta, 2009, p. 35)

Por su parte, Espinosa (2009) considera que la dinámica familiar actual está sometida a presiones, demandas y estrés, tanto en el plano intrafamiliar como en los producidos en otros subsistemas interrelacionados. Plantea que los conflictos intrafamiliares se deben a la falta de conocimiento de adecuadas alternativas de relación, a la incapacidad de un manejo conveniente de las emociones y a un sistema de creencias estereotipadas en cuanto al género o la crianza. También influye la presencia de sentimientos de impotencia y devaluación ante un contexto lleno de dificultades económicas, sociales y políticas:

En la época actual se observa poca claridad en las reglas en varios niveles. Por ejemplo, los padres se sienten incompetentes para establecer reglas firmes con los hijos, debido entre otras cosas, a la influencia de los medios de comunicación masiva (radio, televisión, revistas e internet), que los confunde y los hace sentir "malos padres" si ejercen la autoridad. Asimismo, afloran sentimientos de culpabilidad por dejar a los hijos solos debido a las actividades laborales o por haber vivido una separación, lo que condiciona a una jerarquía ineficaz o se produce una relación amigos/padres donde los hijos no tienen una guía y deben ser más bien un soporte emocional de sus propios padres (Espinosa, 2009, p. 56) 
El resultado para la misma autora, es que, tanto padres como hijos lucen confundidos, desorientados, ineficaces e infelices. Señala que las familias actuales con niños y niñas en edad preescolar y primaria, experimentan grandes demandas y presiones económicas que obligan al padre y la madre a asumir largas jornadas laborales. Por su parte, los infantes permanecen solos, en lugares de cuido o bajo la custodia de otras personas de la familia extensa.

De acuerdo con Arès (2002), los impactos socioeconómicos y sociopolíticos impuestos por la globalización, han diversificado la estructura, el funcionamiento y la tipología de la familia nuclear tradicional:

Hasta hace pocas décadas se hablaba que la familia era la unión legal entre un hombre y una mujer que se unen, con el objetivo de procrear, educar los hijos, y satisfacer necesidades humanas de unión y compañía. Ninguna definición de este tipo se corresponde con los cambios actuales. La unión puede ser legal o no, puede ser para toda la vida, pero las separaciones y los divorcios son frecuentes, los miembros de la unión ya no siempre son heterosexuales, la unión ya no es solo para procrear. Con ello queremos decir que actualmente estamos ante el fenómeno de la diversidad y complejidad familiar. (Arès, 2002, p. 107)

\subsection{Familia y crianza}

De acuerdo con Arès (2002) los modelos asumidos en el pasado ya no corresponden con las exigencias del cambio, pero siguen siendo aprobados como naturales, generando, entre otras cosas, dificultad para establecer límites, confusión de vivir por los hijos y/o a través de ellos, en lugar de con ellos, porque el amor se visualiza como posesión y propiedad:

Tenemos un asignado cultural de maternidad-paternidad que conspira con la formación y educación del ser social al que aspiramos en nuestra sociedad. Creativo, autónomo, con elevado compromiso social, con proyectos propios, solidario, con elevado sentido del deber y responsabilidad. En el caso de las madres, es común que se suela naturalizar un modelo de madre sacrificada y abnegada, que adopta actitudes de sobreprotección, [mimo] excesivo, tolerancia extrema, por lo tanto promotora de hijos dependientes y demandantes. Lo que se justifica mediante frases como: “...así son las madres". Por otra parte, esto conlleva a sentir a los hijos como posesiones, hablando en términos de propiedad, "me saca malas notas". (Arès, 2002, p. 24) 
La misma autora propone redimensionar los roles de padre y madre para los tiempos actuales, ya que no están acordes a las exigencias de cambio y se presenta una contradicción entre los mandatos sociales y la posibilidad real de concretar en la práctica, así como la puesta de acuerdo entre los adultos. Por esto, propone reaprender la crianza, aprender a decir, hacer y sentir lo pertinente y necesario bajo nuevas pautas:

Se deben aprender modelos de cooperación, colaboración, que permitan un fondo de tiempo disponible para la crianza y que garanticen la posibilidad de reemplazo de los adultos en la familia. Reevaluar pautas de crianza, criterios de autoridad, del papel y lugar de los hijos, de conceptos como crecer, la comunicación, las contradicciones básicas que se viven hoy en la tarea de educar. (Arès, 2002, p. 26)

\subsection{Funciones familiares}

Para Zaldívar (2005) las principales funciones de la familia pueden sintetizarse en cuatro: función biosocial, función económica, función cultural y afectiva, y función educativa. A su vez, el funcionamiento familiar saludable cumple exitosamente con seis objetivos que le están histórica y socialmente asignados. Primero, la satisfacción de las necesidades afectivoemocionales y materiales de sus miembros. Segundo, la transmisión de valores éticos y culturales. Tercero, la promoción y facilitación del proceso de socialización de sus miembros. Cuarto, el establecimiento y mantenimiento de un equilibrio que sirva para enfrentar las tensiones que se producen en el curso del ciclo vital. Quinto, el establecimiento de patrones para la convivencia social, y sexto, la creación de condiciones propicias para el desarrollo de la identidad personal y la adquisición de la identidad sexual.

\subsubsection{Familia y establecimiento de patrones para la convivencia social}

De acuerdo con los planteamientos del Ministerio de Educación Nacional de Colombia (2005), las elecciones que realiza la familia sobre cómo desempeñar la práctica socializadora, no son azarosas. Los aspectos culturales son el referente que marca la interacción familiar, ya que permite fortalecer modelos o patrones de comportamientos relevantes para la convivencia, o, por el contrario, otros que conducen al desarrollo de personas sumisas o violentas.

Asimismo, es en el seno familiar en donde niños y niñas empiezan a desarrollar competencias que generan prácticas proclives a la convivencia, como la capacidad para 
manejar sentimientos y emociones, o para tramitar adecuadamente los conflictos. Se aprenden y se ensayan habilidades que implican, entre otras, cooperación, trabajo en equipo, iniciativa, creatividad y autorregulación. En el proceso formativo de la infancia, se observan e imitan los comportamientos y actitudes de las personas adultas, se aprenden formas de vincularse, niveles de tolerancia, estilos de comunicación, cómo resolver conflictos y formas de manejar el enojo (Ministerio de Educación Nacional de Colombia, 2005).

De acuerdo con Rodríguez (2006), es en la familia más que en ningún otro eslabón social, donde los y las infantes aprenden sobre el diálogo, la tolerancia, la solidaridad, el respeto a los derechos humanos y la búsqueda de la justicia, entre otros. Por lo tanto, el grupo familiar constituye un escenario idóneo para aprender estrategias de convivencia. Estas estrategias deben, a su vez, favorecer la creación de ambientes que permitan establecer relaciones no violentas disminuyendo la intensidad de los conflictos, anulando la aplicación de castigos físicos, y mejorando la autoestima y la seguridad personal. Además, señala el autor, los aprendizajes obtenidos en el grupo familiar, posteriormente se multiplicarán en el intercambio que los individuos mantengan con otras instancias sociales.

Para el investigador Tuvilla (2004) la familia, como una de las esferas en que se ejerce la ciudadanía, debe contar con las competencias necesarias para ser capaz de promocionar entre sus integrantes valores y prácticas democráticas como una forma de vida, y así, poder construir una nueva cultura fundada en el comportamiento pro-social, con un modo diferente de pensar y actuar. Sugiere que el grupo familiar es una pieza clave en un mundo que aspira hacer efectivo el derecho humano a la paz, por lo que debe educar en la solidaridad, que implica sentirse responsable de todas las personas:

La solidaridad es un requisito que ayuda a vivir mejor unos con otros, en un encuentro necesario y libre, en el que cada cual gracias a la cooperación, el desinterés y la generosidad, ofrece lo mejor de sí para el bien de la comunidad, a la vez, que desarrolla todas sus potencialidades. (Tuvilla, 2004, p. 232)

A partir de los fundamentos teóricos expuestos, se diseñó la metodología del estudio que se expone en el siguiente apartado. 


\section{Metodología}

Con el objetivo de lograr una percepción más integral y completa del fenómeno de estudio, se seleccionó un enfoque mixto, que implicó un proceso de recolección, análisis y vinculación de datos cuantitativos y cualitativos. Esto permitió explorar distintos niveles del problema seleccionado y obtener una mayor variedad de perspectivas.

La investigación se dividió en cuatro etapas. La primera consistió en la revisión bibliográfica, lo que posibilitó la recopilación de información relevante y necesaria al problema de estudio. Como segundo paso, se utilizó la estrategia metodológica de recopilar información proveniente de formularios de consultas, efectuados al Departamento de Psicología (DP) del centro educativo. Estas consultas las realizaron personas encargadas de la crianza de niños y niñas entre los tres y los diez años de edad, provenientes de un centro educativo ubicado en el área metropolitana, entre los ciclos lectivos 2010 y 2011.

Como resultado de esta revisión, se contabilizaron 226 consultas, planteadas por los siguientes representantes familiares: $64 \%$ por madres de familia, que fueron las mayores consultantes, seguidas por ambos progenitores con un 18\%. En tercer lugar, se encontraron figuras paternas con un $10 \%$; y el restante $8 \%$ estuvo compuesto por otras personas encargadas de la crianza, como abuelos, abuelas y tías.

Estas inquietudes no correspondieron necesariamente a 226 representantes familiares, ya que en el transcurso de los ciclos lectivos 2010 y 2011, algunas personas consultaron varias veces sobre temas distintos.

Es importante explicar que las consultas fueron en su momento expuestas a la psicóloga escolar de manera individual, o acompañada de algunas docentes. En todos los casos se llenó un formulario de consulta propiedad del DP, que posteriormente sirvió de insumo para esta investigación.

En la tercera etapa, se revisaron los 226 formularios de consulta. Toda la información proveniente de estos se sistematizó de acuerdo a los motivos de consulta de las familias, con un total de 29 inquietudes (redactadas como preguntas) en torno a temas de crianza.

Adicionalmente, y en lo que respecta a los datos cualitativos, a partir de la indagación de los 226 formularios se realizó un listado de frases textuales y explicaciones de algunas de las narraciones brindadas por las personas participantes, a fin de ejemplificar los datos cuantitativos y comprender el fenómeno de estudio a mayor profundidad. 
En la cuarta fase del proceso, las 29 preguntas se subdividieron por temáticas, al igual que la información cualitativa, constituyendo un total de cinco categorías de análisis.

Todo esto con el objetivo de indagar sobre las inquietudes y estrategias empleadas en la crianza, y si estas fueron o no compatibles con el aprendizaje de la convivencia.

\section{Análisis de resultados}

Durante los años 2010 y 2011, madres, padres, y otras personas encargadas de niños y niñas entre los tres y los diez años, realizaron 226 consultas al Departamento de Psicología (DP), relacionadas con inquietudes en torno a la crianza.

Para efectos del análisis de los datos, el registro de las 226 inquietudes se sistematizó en 29 preguntas, que se plantearon en referencia a la atención de niños y niñas, tal y como se exponen en la tabla 1.

\section{Tabla 1}

Preguntas que sistematizan las 226 consultas formuladas por familias al DP.

1. ¿De qué forma poner límites?

2. ¿Cómo hacer para que sigan instrucciones de adultos?

3. ¿Cómo aplicar consecuencias de manera efectiva?

4. ¿Cómo saber si son justas las consecuencias que se aplican en el ámbito escolar?

5. ¿Cómo promover tolerancia y autocontrol?

6. ¿Cómo ejercer un rol de autoridad sin ser autoritario?

7. ¿Qué hacer ante situaciones de berrinche?

8. ¿Cómo lidiar con reacciones violentas?

9. ¿Cómo enseñar a enfrentar y solucionar conflictos con otros niños?

10. ¿Cómo hacer para que duerman en su propia cama?

11. ¿Estrategias para que adquieran mayor seguridad en sí mismos?

12. ¿Cómo ayudar a vencer temores?

13. ¿Cómo favorecer la integración social?

14. ¿Cómo promover autonomía?

15. ¿Cómo ayudar a vencer temores nocturnos?

16. ¿Cómo ayudar a fortalecer la autoestima?

17. ¿Cómo explicar conflictos familiares?

18. ¿Cómo explicar cuando un padre o madre no se involucra en la crianza?

19. ¿Cómo ayudar cuando los padres se separan?

20. ¿Cómo ayudar a enfrentar la muerte de un ser querido?

21. ¿Cómo proceder ante situaciones de angustia ante la separación?

22. ¿Cómo explicar sobre una enfermedad grave de un ser querido?

23. ¿Cómo explicar problemas económicos?

24. ¿Cómo apoyar en las dificultades académicas?

25. ¿Cómo manejar la indisposición para asistir a la escuela?

26. ¿Cómo motivar en el estudio?

27. ¿Cómo enseñar a valorar lo que se tiene?

28. ¿Qué hacer cuando no dicen la verdad?

29. ¿Cómo enseñar a compartir?

Fuente: Elaboración propia 
Posteriormente, las 29 preguntas se subdividieron en temáticas, conformando cinco grandes categorías de análisis. En la tabla 2 se presentan las dimensiones sobre las que consultaron representantes familiares.

\section{Tabla 2}

Categorías en las que se agrupó la sistematización de las consultas.

\section{Categoría}

1. Inquietudes relacionadas con aspectos disciplinarios.

2. Inquietudes relacionadas con habilidades sociales.

3. Inquietudes relacionadas con cambios o conflictos en la dinámica familiar.

4. Inquietudes relacionadas con aspectos de desempeño académico.

5. Inquietudes relacionadas con valores.

Fuente: Elaboración propia

En el gráfico 1, se aprecian las cinco categorías en que se resumieron las consultas, con su respectivo porcentaje de consulta.

\section{Gráfico 1}

Consultas por categorías, realizadas por familias al Departamento de Psicologia en los ciclos lectivos 2010 y 2011

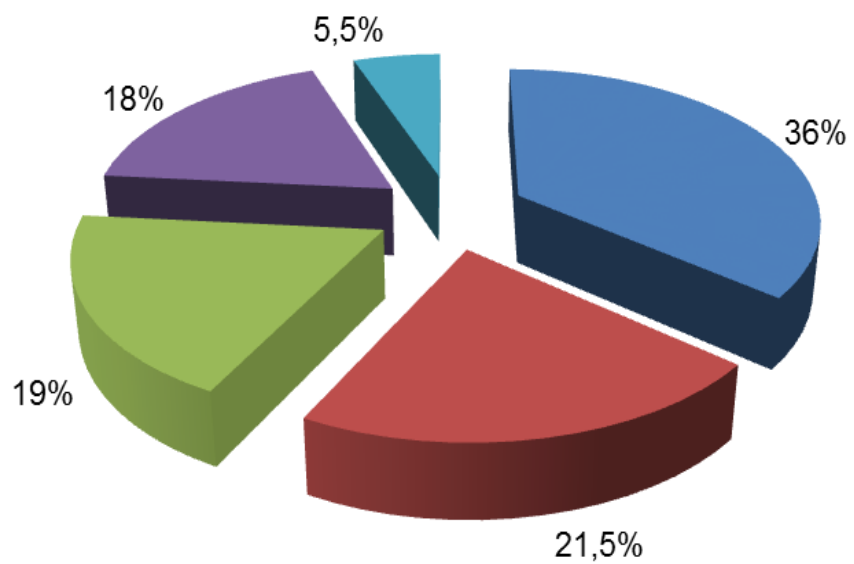

$$
\begin{aligned}
& \text { - ASPECTOS DISCIPLINARIOS } \\
& \text { - CAMBIOS Y CONFLICTOS } \\
& \text { - IMPLEMENTAR HABILIDADES } \\
& \text { - ASPECTOS ACADEMICOS } \\
& \text { - PROMOVER VALORES }
\end{aligned}
$$

Fuente: Elaboración propia 
A continuación, se presenta el análisis de resultados por categoría, iniciando con la tabla 3, que muestra las preguntas y los porcentajes respectivos a la primera dimensión.

\section{Tabla 3}

Categoría \#1: Inquietudes relacionadas con aspectos disciplinarios.

Preguntas (Todas las preguntas están referidas a los niños y las niñas.) $\%$ de consultas

¿De qué forma poner límites? $10 \%$ ¿Cómo hacer para que sigan instrucciones de adultos?

$6.0 \%$

¿Cómo aplicar consecuencias de manera efectiva?

$5.0 \%$

¿Cómo saber si son justas consecuencias que se aplican en el ámbito escolar?

$4.0 \%$

¿Cómo promover tolerancia y autocontrol?

$3.5 \%$

¿Cómo ejercer un rol de autoridad sin ser autoritario?

$2.6 \%$

¿Qué hacer ante situaciones de berrinche?

$2.6 \%$

¿Cómo lidiar con reacciones violentas?

$2.2 \%$

Porcentaje categoría \#1: $\quad 36 \%$

Fuente: Elaboración propia

Del total general de consultas, un $36 \%$ de las personas planteó inquietudes con respecto al tema de la implementación de la disciplina en la dinámica familiar, identificando la indisciplina como una fuente común de desacuerdos entre personas adultas e infantes. Aunado a esto, la indisciplina fue interpretada como un inconveniente para el logro de la convivencia familiar. En tanto que el concepto subyacente de convivir no incluye el desacuerdo como parte de la interrelación: “...que no hagan caso (hijos) me vuelve loca, yo quiero ser como esas familias que conviven siempre felices". En otras palabras, para las personas participantes, la convivencia se entiende como una situación de armonía que excluye el desacuerdo, cuando, por el contrario, debe ser un aprendizaje del respeto a la diversidad, en que los conflictos y su resolución alternativa a la violencia, son experiencias valiosas.

En la revisión de los expedientes escolares del DP, se evidenció que las personas responsables de la crianza presentan poca claridad sobre etapas del desarrollo infantil, cómo brindar instrucciones, establecer límites, ejercer la guía adulta, y enfrentar berrinches y situaciones violentas, plantear y cumplir consecuencias, y promover el auto control.

Sobre el conocimiento acerca de las etapas del desarrollo infantil, se determinó:

- Dificultad para identificar conductas esperadas y no esperadas en diferentes etapas del desarrollo.

- Dificultad para establecer límites o consecuencias distintas entre el grupo de hermanos o hermanas (de acuerdo a la situación y a las etapas del desarrollo). 
- Dificultad para realizar cambios en torno a la disciplina conforme los niños y las niñas evolucionan.

Sobre la comunicación de instrucciones y puesta de límites, se encontró:

- Poca claridad sobre las palabras a emplear para lograr que se cumpla una instrucción.

- Sugirieron emplear un tono de voz fuerte "para que se escuche".

- Recurren a la repetición constante de la instrucción, sin lograr el efecto deseado.

- Reportaron experimentar enojo, frustración y/o impotencia al percatarse de que la criatura no cumple con lo solicitado.

- En algunos casos, cuando no logran que las personas menores de edad cumplan la demanda solicitada, las personas adultas la ejecutaron impartiendo regaños y/o reclamos.

- El cansancio conduce a cambiar lo solicitado: “... ya agotada yo le digo, bueno al menos recoja dos y yo recojo lo que queda, pero ni así quiere hacerlo."

Sobre ejercer la guía adulta se presentó:

- Poca disposición de tiempo para la crianza, lo que genera culpa y conduce a que se toleren algunas conductas: “...yo sé que eso está mal, pero no me animo a quitárselo, porque casi ni lo veo para estar pelee que pelee el ratito que estamos juntos".

- Consideraron que la persona adulta debe mostrarse molesta, "al menos seria" para ser identificada como autoridad.

- Señalaron situaciones en que niños y niñas son los que ejercen la autoridad: “...ella se cree como una reina y le da órdenes a todos, sino hacemos lo que quiere llora y da gritos”, “... un día hasta nos echó de la casa y llamó a los abuelos para acusarnos”.

Sobre el enfrentamiento de berrinches y situaciones violentas, se registró:

- Las escenas de berrinches y/o reacciones violentas fueron reportadas como las más difíciles de manejar, en especial cuando ocurren en escenarios públicos: “...la verdad es que yo le doy lo que pide para que se calle, todo el mundo me vuelve a ver y a mí se me cae la cara de la vergüenza”, “...cuando se enoja es terrible, da patadas al que se le acerca, hasta que sudo tratando de calmarla". Las personas adultas ceden a las solicitudes o no cuentan con estrategias para intervenir. 
Sobre plantear y cumplir consecuencias se encontró:

- Como primera acción, implementan el regaño constante, aún cuando pocas veces corroboran su efectividad.

- La mayoría de las consecuencias no tienen relación con las etapas de desarrollo o se aplican a niños y niñas con edades y características distintas.

- Efectúan amenazas poco sensatas que no cumplen: "Si no viene ya, ahí lo dejo solo") y que no corresponden con la falta.

- Con frecuencia cambian o incumplen la consecuencia previamente establecida: “...bueno, solo por esta vez se lo compro, es la última”.

- En algunos casos, el castigo físico es empleado como una solución rápida y efectiva para enseñar al infante "quien manda": "... si es mucho va el manazo y solo así se calma y me respeta".

Sobre promover auto control se determinó:

- Las personas encargadas de la crianza exteriorizaron deseos por enseñar tolerancia y autocontrol, pero expresaron no saber cómo lograrlo.

- El modelaje de las personas adultas no fue planteado como alternativa. Tampoco el aprendizaje de algunas habilidades sociales como el reconocimiento de las propias emociones y de las de los demás.

En todos los casos, las familias relacionaron las dificultades disciplinarias con un clima de tensión, enojo, mal humor y distanciamiento afectivo entre los integrantes. Además de un incremento en la sensación de que la crianza es una labor muy difícil de desempeñar, lo que condujo a que buscaran orientación del personal del centro educativo. Sin embargo, cuando la institución escolar fue la que aplicó consecuencias, las personas cuidadoras manifestaron desconfianza e inconformidad y un deseo por justificar la conducta del infante.

Las familias del estudio no visualizaron la importancia de la adquisición de normas sociales de convivencia como una experiencia de aprendizaje que, posteriormente, niños y niñas puedan trasladar a otras esferas sociales. Por el contrario, modelaron inestabilidad, incongruencia, comunicación poco clara o en base a regaños y el uso de la violencia para solucionar desacuerdos. 
Con respecto a la segunda categoría, en torno a las habilidades sociales que se desean fortalecer en los niños y niñas para que adquieran más seguridad, mayor autoestima y más autonomía (y, por lo tanto, superen entre otros temores), se ubicó un $21.5 \%$ del total de consultas, que se describe en la tabla 4.

\section{Tabla 4}

Categoría \#2: Inquietudes relacionadas con habilidades sociales.

\begin{tabular}{lc}
\hline Preguntas (Todas las preguntas están referidas a los niños y las niñas.) & \% de consultas \\
\hline ¿Cómo enseñar a enfrentar y solucionar conflictos con otros niños? & $8.0 \%$ \\
¿Cómo hacer para que duerman en su propia cama? & $2.8 \%$ \\
¿Estrategias para que adquieran mayor seguridad en sí mismos? & $2.6 \%$ \\
¿Cómo ayudar a vencer temores? & $2.2 \%$ \\
¿Cómo favorecer la integración social? & $2.2 \%$ \\
¿Cómo promover autonomía? & $2.2 \%$ \\
¿Cómo ayudar a vencer temores nocturnos? & $0.9 \%$ \\
¿Cómo ayudar a fortalecer la autoestima? & $0.4 \%$ \\
\end{tabular}

Fuente: Elaboración propia

La principal inquietud que experimentaron las personas encargadas de la crianza, giró en torno a enseñar a los niños y niñas a resolver conflictos fuera del núcleo familiar, especialmente en el escolar. Una madre narró: “...a mí me da una gran tristeza que otra compañera la maltrate y le diga cosas, es que ella no sabe defenderse porque pasa entre adultos que la chinean". No lograron precisar la importancia de favorecer habilidades sociales que eduquen la convivencia, como la comunicación asertiva, la escucha, la negociación, la empatía, el respeto a la diversidad. Por el contrario, el empleo de la violencia para la autodefensa surgió como válido: “... le voy a contar que el papá ya le dio permiso para que le devuelva un buen golpe al que intente golpearlo... Hasta practica con él luchitas para que aprenda a defenderse".

El tema de la autonomía también cobró relevancia, asociado a incongruencias sobre la conveniencia o no de favorecer el crecimiento. Por una parte, las personas cuidadoras esperan que la criatura se desenvuelva con seguridad, sin temor y tenga autonomía sobre su cuerpo (ej. bañarse o vestirse por sí mismo), sus pertenencias y espacios (ej. dormir en su propio cuarto); pero, por otra, experimentan sentimientos que afectan la independencia: “... uno es tonto y piensa que si se hacen grandes como que ya no lo van a buscar y querer, más lindo así pequeñitos". 
El tiempo dedicado a la crianza también fue mencionado como un paralizador de la autonomía: “...creo que todo este tiempo lo hemos dejado dormir en nuestra cama, porque a uno le da lástima lo poquillo que estamos con él, entonces uno piensa que aunque sea compartir durmiendo".

A las familias del estudio les preocupó cuando los niños y niñas muestran poca seguridad en sí mismos, se les dificulta la integración con otros pares y/o la autoestima se ve afectada. Sin embargo, no establecen una relación directa para favorecer la autonomía u otras habilidades sociales, ni cómo ambas son dimensiones esenciales del convivir.

Percibieron a los menores de edad desde un rol de víctimas del maltrato de terceros, como personas indefensas a las que deben proteger. Prevaleció el término pobrecito: “... a mí me dio una lástima que ese chiquillo le pegara, pobrecito, yo hasta lloré de la cólera". Niños y niñas no son educados para el desarrollo de su inteligencia emocional, por lo tanto, no saben resolver situaciones de conflicto de forma alternativa a la violencia, o quedan a la espera de la sobreprotección de figuras adultas. No se les enseña a ser proactivos, ciudadanos que conviven entre sí y constructores de una cultura de paz.

En lo concerniente a la tercera categoría, cambios o situaciones de conflicto en la estructura familiar, se situó un 19\% del total de consultas, como se representa en la tabla 5.

\section{Tabla 5}

Categoría \#3: Inquietudes relacionadas con cambios o conflictos en la dinámica familiar.

\begin{tabular}{lc}
\hline Prequntas (Todas las preguntas están referidas a los niños y las niñas.) & $\%$ de consultas \\
\hline ¿Cómo explicar conflictos familiares? & $5 \%$ \\
¿Cómo explicar sobre el padre o madre que no se involucra en la crianza? & $4 \%$ \\
¿Cómo ayudar cuando los padres se separan? & $2.6 \%$ \\
¿Cómo ayudar a enfrentar la muerte de un ser querido? & $2.6 \%$ \\
¿Cómo proceder ante situaciones de angustia en la separación? & $1.7 \%$ \\
¿Cómo explicar sobre una enfermedad grave de un ser querido? & $1.8 \%$ \\
¿Cómo explicar problemas económicos? & $1.3 \%$ \\
& \\
& Porcentaje categoría \#3: \\
\end{tabular}

\section{Fuente: Elaboración propia}

Entre quienes consultaron prevaleció la creencia de que la infancia debe estar excluida de situaciones de sufrimiento, lo que explica que en muchas ocasiones se mantenga a los menores al margen de los acontecimientos y/o solo se atiendan dudas específicas. En otros casos, se dieron explicaciones no coincidentes con la realidad; también refirieron entretenerlos para promover la distracción y evitar el sufrimiento. 
Las personas encargadas de la crianza participantes de este estudio, afirmaron desconocer cómo comunicar a niños y niñas situaciones de cambio, conflicto, enfermedad o muerte. No tenían claridad sobre las respuestas que debían dar en relación a la circunstancia y/o la etapa de desarrollo; tampoco lo adecuado o no de referirse a los hechos. Evidenciaron que en su propio proceso socializador, no se les enseño a conocer, nombrar y expresar emociones, lo que dificulta acompañar a las criaturas en estos procesos: “...yo paso llorando por mi papá, no quiero que mi hijo me vea, pero se me salen las lágrimas, le digo que es por lo que están dando en la tele que me da tristeza, es que no sé cómo explicarle".

La poca trascendencia que se le brinda al desarrollo de la inteligencia emocional y al de las habilidades sociales, influye en aspectos determinantes de la crianza, como la posibilidad de exteriorizar sentimientos y de ser contenido por las personas mayores. Esta situación, a su vez, afecta el aprendizaje de la convivencia en el núcleo familiar. Niños y niñas no son preparados para enfrentar cambios y pérdidas, por lo que son vividas a través de información inconsistente, sin conexión con los sentimientos respectivos.

Otra creencia vigente entre consultantes, fue la de la "familia ideal" (padre- madre-hijos). En las situaciones de separación de la pareja de progenitores, existió la creencia de que las criaturas se encuentran en desventaja y se genera un gran sentimiento de culpa que obstaculiza otros planos de la relación: “...desde que nos separamos, yo ando como en otras, entonces lo dejo que haga y deshaga ya no lo regaño porque pobre". Las separaciones son vividas a través de incongruencias entre lo que se dice, se hace y se siente: “...a mi ex yo no lo soporto, pero que me queda, decirle a los chiquitos que él es un amor para que no sufran, pero bueno ellos mismos se dan cuenta de que es un irresponsable con la plata". Se presentó como una situación común la sensación de estar "en deuda" con los niños y niñas y tratar de compensar vacíos emocionales con la adquisición de bienes materiales: “....cuando se la lleva el tata, le compra de todo, viene toda exigente conmigo, es que él quiere comprarla".

Con respecto al tema de la convivencia familiar, las situaciones de conflicto y de cambio no fueron contempladas como oportunidades para enseñar sobre comunicación asertiva, diversidad de criterios, negociación, congruencia entre sentimientos y acciones, el proceso de la vida y la muerte, entre otras. Las experiencias de cambio y/o desacuerdos fueron desaprovechadas como experiencias del convivir. Por el contrario, las familias participantes las vivieron desde la impotencia, la frustración, el enojo, la tristeza solapada, la incongruencia, la lástima y la culpa. 
La cuarta categoría de consultas agrupó inquietudes sobre aspectos académicos como el apoyo escolar, la desmotivación y las dificultades académicas; están representadas por un $18 \%$, tal y como se ilustra en la tabla 6.

\section{Tabla 6}

Categoría \#4: Inquietudes relacionadas con aspectos del desempeño académico. Preguntas (Todas las preguntas están referidas a los niños y las niñas.) $\quad \%$ de consultas ¿Cómo ayudar en las dificultades académicas? $12 \%$ ¿Cómo manejar la indisposición para asistir a la escuela? ¿Cómo motivar en el estudio?

$3 \%$

$3 \%$

Porcentaje categoría \#3: $18 \%$

\section{Fuente: Elaboración propia}

Es importante señalar que la alta incidencia de inquietudes en esta categoría, respondió de manera directa a que la población consultante se encontraba en un centro educativo. Adicionalmente, se encontró que el éxito escolar fue vivido como personal y como signo del "buen apoyo en el hogar". En otras palabras, una forma de obtener reconocimiento "de buen padre/ madre/ cuidador" es a través del rendimiento escolar de la criatura, por lo que un $18 \%$ formuló preguntas sobre cómo brindar apoyo en el desempeño académico.

Sin embargo, la mayoría de las inquietudes obedecieron más al deseo de control y/o sobreprotección que al de desarrollar en el infante autonomía, responsabilidad y autoregulación. En muchos casos, los límites entre el rol de progenitor y el del niño o niña como estudiante fueron difusos: “...yo sé que no soy la que está en la escuela, pero si mi hijo no cumple con algo, me siento irresponsable, me preocupo como si fuera a mí a la que la maestra va a regañar", "...cuando llega un recado o una boleta me pongo toda nerviosa, se me altera el día, además el chiquito me dice que no fue así, y ya no sé a quién creerle, entonces llamo a la maestra por teléfono para que me explique".

Las personas responsables de la crianza duplicaron sus funciones y asumieron como propias demandas escolares, experimentando cansancio y frustración:”...jes el colmo!, son las diez de la noche y yo ahí ayudándole a pintar para que no lleve tan fea la tarea”, “...todas las mañanas inician con un pleito porque no se quiere meter al baño y después viene en el carro llorando que no quiere ir al kínder, tengo que venir convenciéndolo, pero es muy cansado”.

Desde la promoción de la convivencia, algunas habilidades sociales como la autonomía, y valores como el esfuerzo, la perseverancia, la responsabilidad, son poco vivenciados en la 
interrelación familiar. Como resultado, la crianza no se disfruta y resulta para la población consultada una labor desbordante.

La última categoría del total de consultas agrupó justamente las inquietudes relacionadas con el fortalecimiento de valores, que implicaron un $5.5 \%$ de los datos, tal y como se presentan en la tabla 7.

\section{Tabla 7}

Categoría \#5: Inquietudes relacionadas con valores.

Preguntas (Todas las preguntas están referidas a los niños y las niñas.) $\%$ de consultas

¿Cómo enseñar a valorar lo que se tiene? $2.2 \%$ ¿Qué hacer cuando no dicen la verdad?

$2.2 \%$

¿Cómo enseñar a compartir?

$0.9 \%$

\section{Porcentaje categoría \#5: $\quad 5.5 \%$}

\section{Fuente: Elaboración propia}

Específicamente en este rubro, el grupo participante en el estudio reveló conocer la importancia de los valores, pero ignorar estrategias efectivas para promoverlos. Se autopercibieron impotentes frente al consumismo que los rodea: “...me da miedo verlo tan materialista, quiere de todo, no sabe que las cosas cuestan".

Las figuras familiares como modelos de la vivencia de valores, no fueron sugeridas como alternativa a imitar, tampoco para el desarrollo de algunas habilidades sociales como la empatía o el interés en el entorno.

La formación en valores fue una labor encomendada a otra instancia social, la escuela: “...nosotros escogimos esta escuela por lo de los valores que queremos inculcarle, le cuesta mucho compartir y sabemos que ustedes le están enseñando”, “...bueno mi hija estuvo muy mentirosa, yo hablé con la maestra y ella hizo como una dinámica y desde entonces ha cambiado".

Una dimensión de plena trascendencia para la enseñanza de la convivencia, la formación en valores, quedó fuera de la esfera familiar, y su aprendizaje fue encomendado a la siguiente instancia social.

\section{Conclusiones}

La familia actual, entendida como una multiplicidad de tipologías familiares, producto de la globalización, continúa siendo un eslabón clave en la promoción del convivir, una esperanza en un mundo que aspira a hacer efectivo el derecho humano a la paz. 
Para el logro de esta tarea, la estructura familiar necesita estar fortalecida y ser capaz de responder a situaciones distintas y cambiantes. Al respecto, plantea Zaitegi (2008) que el seno familiar, a través de la socialización, debe procurar favorecer competencias y habilidades para vivir con dignidad, destacando el autoconocimiento, la autorregulación y la capacidad de resiliencia. Con respecto a las habilidades sociales, refiere el mismo autor aquellas para relacionarse positivamente con otras personas, como cooperar, negociar y trabajar en equipo, entre otras.

Los resultados de este estudio precisaron una situación muy distinta a la planteada por Zaitegi. Se evidenciaron cambios en la estructura, dinámica e interrelación familiar, que inciden negativamente en las competencias para la crianza y, simultáneamente, en el deterioro del aprendizaje de la convivencia. Los padres y las madres participantes se mostraron tensos, agotados, frustrados e incluso desbordados, al intentar cumplir con la multiplicidad de funciones socialmente asignadas. Se presentó una contradicción y una gran distancia entre los mandatos sociales y la posibilidad real de concretarlos en la práctica.

Entre las familias del estudio, la comunicación dirigida a niños y niñas estuvo sujeta a espacios limitados de tiempo y regida por regaños. Los patrones comunicativos no fueron directos ni claros. En muchos casos, se orientaron a evadir conflictos o sentimientos. Se entorpeció el aprendizaje de la escucha, también el respeto a la opinión ajena, la resolución del conflicto a través de la palabra y la posibilidad de pensar distinto y plantearlo. Todos estos, elementos claves de la convivencia.

En el ejercicio de la crianza, prevaleció un sentimiento de inseguridad y culpa que conlleva a la sobreprotección y la tolerancia excesiva, las cuales, posteriormente, impiden realizar los desprendimientos necesarios para crecer con libertad y autonomía.

En lo que respecta a los aspectos disciplinarios, se evidenció que un gran porcentaje de las familias del estudio establecieron límites poco claros, o rígidos y difusos, invadiendo los espacios y fracturando los procesos de individuación. Sobre este asunto, la investigadora Espinosa (2009) explica que las figuras parentales se sienten incompetentes en el establecimiento firme de reglas, porque temen ser vistos como "malos padres" si ejercen la autoridad.

Algunas personas participantes del estudio recurrieron a los castigos físicos, indicando que después del golpe el "niño entiende" e identifica "quien manda". Al respecto, Henao (2005) ha encontrado evidencias que señalan una tendencia a reproducir por fuera del hogar 
la violencia vivida en la familia. Refiere que todo acto de violencia constituye una vulneración a los derechos y las libertades fundamentales de las personas y una ofensa a su dignidad.

Los niños y las niñas, contrario al aprendizaje de la convivencia, copian los modelos violentos, que se presentan como válidos en la resolución de conflictos y los reproducen en el contexto familiar y, posteriormente, en otras instancias sociales.

Sánchez (2009) señala que el recurso de la empatía se dificulta cuando los castigos aplicados responden más a una reacción emocional irreflexiva y sin explicación lógica. Indica que los castigos tienen implicaciones en la relación de apego, puesto que se produce un aprendizaje, pero por distanciamiento. Esto debido a que se trata de un estímulo aversivo que, como tal, produce rechazo y malestar emocional, con implicaciones negativas en el desarrollo de la personalidad. Esta situación, según Zaldívar (2005), dificulta la cohesión familiar en términos de los vínculos emocionales, la cual es una dimensión central en la socialización e interacción familiar.

Además, para López (2005) las diversas demandas, presiones sociales y multiplicidad de tareas que deben asumir las figuras adultas, interfieren en esta cohesión grupal, menoscabando la función socializadora y, específicamente, el aprendizaje de la convivencia. Este aprendizaje fue delegado a la escuela, tal y como lo advirtieron familias participantes.

Rodríguez (2006) recuerda que la familia, más que ningún otro eslabón social, debe ser el lugar donde los niños y las niñas aprendan sobre el diálogo, la tolerancia, la solidaridad, el respeto a los derechos humanos y la búsqueda de la justicia, entre otros, por lo que se hace imprescindible dotarla de insumos para que esta labor prevalezca.

Por su parte, Arès (2009) propone revisar los mandatos sociales a los que está sujeta la familia como primera instancia socializadora, a fin de fortalecerla. Propone reaprender las pautas de crianza, redimensionar los roles, en especial de padre y madre, aprender modelos de cooperación y colaboración, evaluar los criterios de autoridad del papel y lugar de los hijos, y conceptos como crecimiento y comunicación así como las contradicciones básicas que se viven en la tarea de educar.

En el mismo sentido, Rentería, Lledios, y Giraldo (2008) plantean la necesidad imperiosa de contribuir a que la familia se convierta en un escenario que se pueda reconstruir y en el que sea factible enseñar y practicar la convivencia. Un espacio donde se desarrollen competencias para al descubrimiento del otro y de sí mismo, como fundamento para el 
desarrollo de la empatía, asertividad, autonomía, y la posibilidad de valorar y respetar las diferencias.

En esta línea de pensamiento, la Organización para las Naciones Unidas (1994) propone que implicar a las familias en la educación pacificadora requiere desarrollar formas de pensamiento crítico ante la sociedad, y reflexionar y asumir valores, normas y actitudes facilitadoras de la convivencia y capaces de dar respuesta a los problemas que se les plantean diariamente. Para ello, es imprescindible reconocer y aceptar los valores que existen en la diversidad de los individuos, los sexos, los pueblos y las culturas, así como desarrollar la capacidad de comunicar, compartir y cooperar con los demás.

En síntesis, de los resultados expuestos surge la urgencia de trabajar en procesos reflexivos y educativos con las familias costarricenses, a fin de encontrar estrategias que no solo den soluciones a las inquietudes en torno a la crianza, sino que fortalezcan el protagonismo de las familias como gestoras en la construcción de una cultura de paz. Para ello, se requiere concebir la promoción de la convivencia no solo como un factor de bienestar para las personas, sino como la base desde la que se construyen la ciudadanía, la calidad de un país en el futuro y también la posibilidad de entendimiento entre naciones.

\section{Referencias}

Arès, Patricia (2002). Psicología de Familia. Una aproximación a su estudio. La Habana, Cuba: Editorial Félix Varela.

Arès, Patricia. (2009) Conferencia de la Dra. Patricia Arès Muzio. Recuperado de http://www.ipes.anep.edu.uy/documentos/pos grados/conferencias/ares.pdf

Chile, Ministerio de Educación. (2008). Estudio Nacional de Convivencia Escolar. Santiago, Chile: MINEDUC.

Colombia, Ministerio de Educación Nacional. (2005). Política Educativa para la formación escolar en la convivencia. Recuperado de http://www.mineducacion.gov.co/1621/articles-90103 archivo pdf.pdf

Espinosa, María. (2009). Panorama de las relaciones familiares en la época actual. En Marco Murueta y Maricela Osorio (Eds.), Psicología de la familia en países latinos del siglo XXI (pp.43-60). México D.F.: Asociación Mexicana de Alternativas en Psicología.

Henao, Juanita. (2005). La prevención temprana de la violencia: una revisión de programas y modalidades de intervención. Bogotá, Colombia: Sin editorial. 
López Jesús. (2005). Proyecto Educativo para la mejora de la convivencia. Revista Currículum y Formación del profesorado, 9(1). Recuperado de http://www.ugr.es/ recfpro/rev91COL3.pdf

Murueta, Marco. (2009). Familia y proyecto social en la teoría de la praxis. En Marco Murueta y Maricela Osorio (Eds.), Psicología de la familia en países latinos del siglo XXI (pp. 2342). México D.F.: Asociación Mexicana de Alternativas en Psicología.

Murueta, Marco. Osorio, Maricela. (2009). Psicología de la familia en países latinos del siglo XXI. México D.F.: Asociación Mexicana de Alternativas en Psicología.

Organización para las Naciones Unidas. (1994) Carta de las Naciones Unidas y Estatuto de la Corte Internacional de Justicia. Recuperado de http:// www.un.org/es/documents/charter/

Rentería, Erico, Lledios, Esperanza, y Giraldo, Alba. (2008). Convivencia familiar como lectura aproximativa desde elementos de la psicología social. Revista Psicología Social 4, 42744.

Rodríguez, Bertha. (2006). Los métodos alternativos de solución de conflictos: una estrategia inteligente para facilitar la convivencia pacífica. Revista Trabajo Social, 3, 124-139.

Sánchez, Ginette. (2009). Teorías de niños y niñas sobre el castigo parental aportes para la educación y la crianza. Revista Actualidades Investigativas en Educación, 9, 1-29. Recuperado de http://revista.inie.ucr.ac.cr/buscararticulos/controlador/Article/accion/show/articulo/teorias-de-ninos-y-ninas-sobre-el-castigoparental-aportes-para-la-educacion-y-la-crianza.html

Tuvilla, José. (2004). Cultura de Paz. España: Editorial Descléer de Brouwer, S.A.

Zaitegi, Nélida. (2008) Competencias para una vida nueva. Santiago, Chile: Sin editorial.

Zaldívar, Dionisio. (2005). Funcionamiento Familiar Saludable. Recuperado de http://www.sld.cu/galerias/pdf/sitios/rehabilitacion-temprana/funcionamiento familiar.pdf 